\title{
Adsorption and Precipitation of Organic Phosphonates onto the Calcite Stepped Surfaces
}

\author{
Wu LEI ${ }^{1, a}$, Chun-Yu CHEN ${ }^{2, b,{ }^{*},} \mathrm{Ni} \mathrm{BAI}^{2, \mathrm{c}}$ \\ ${ }^{1}$ Institute of Industrial Chemistry, Nanjing University of Science \& Technology, Nanjing 210094 , \\ China \\ ${ }^{2}$ School of Metallurgy and Materials Engineering, Jiangsu University of Science and Technology, \\ Zhangjiagang 215600, China \\ aleiwuhao@mail.njust.edu.cn, bjust_chenchunyu@163.com, ${ }^{c}$ bnbaini@sina.cn \\ ${ }^{*}$ Corresponding author
}

Keywords: Phosphonate, Calcite, Adsorption, Molecular Dynamic Simulation.

\begin{abstract}
The inhibition of calcite growth in the presence of seven phosphonates (AMP DTPMP) was investigated by means of molecular dynamic simulation in industrial water environment. It has been found that the phosphonic molecules can energetically interact well with the calcite (104) stepped surfaces at the stepped dihedral sites. Furthermore, the strength of adsorption is DTPMP > HDTMP > TDTMP > EDTMP > NTMP > NDP > AMP based on the binding energy including strong electrostatic interactions between the oxygen atom in phosphonic functional groups and the calcium ions of the calcite (104) surface, and the formation of hydrogen bond. The results indicate that the phosphonic group number and the length of the backbone methylene chain play an important role in inhibitor effectiveness.
\end{abstract}

\section{Introduction}

The precipitation of calcium carbonate is of fundamental importance in great many researches for instance medicine, industry, and households [1-3]. The research on calcium carbonate particles is mainly determined by the three polymorphs of $\mathrm{CaCO}_{3}$, namely calcite, aragonite and vaterite $[4,5]$. Thereinto calcite is one of the most stable salts in industrial water treatment under normal atmospheric conditions [6]. The (104) surface is by far the most stable plane of calcite and dominates the observed morphology $[7,8]$.

Another area of research is growth inhibition in the presence of both organic and inorganic additives. For example, organic phosphonates are currently important and effective class of inhibitors available in water cooling systems. Phosphonates have a strong tendency to adsorb onto a variety of surfaces, e.g., calcite [9], barite [10] and iron oxides [11]. A considerable amount of researches have focused on the inhibition of growth of the calcite (104) plane, but not much effort has been put into studying the stepped calcite surface. No experimental surface is truly planar, and there are always defects like steps and kinks. Stipp studied the calcite (104) face using scanning force microscopy (SFM) and indicated that steps spread one layer at a time [12]. Hillner and Gratz studied calcite growth by means of atomic force microscopy (AFM) and found that crystal growth occurred through steps and spiral dislocations [13]. Moreover, Nora H. de Leeuw employed atomistic simulation techniques to study the molecular adsorption of water onto the stepped and planar calcite surfaces and found that the stepped planes are good models for growth steps [14].

In previous work, the interaction of three phosphonates with calcite surfaces was examined by molecular dynamic simulation [15] using Materials Studio v3.0 (Accelrys, San Diego, CA) under compass force field [16]. The results showed the $\mathrm{Ca}-\mathrm{O}\left(-\mathrm{PO}_{3}{ }^{2-}\right)$ distance after simulation was less than $2.5 \AA$ in accordance with the length of the factual Ca- $\mathrm{O}$ bond, implying that phosphonates have a high affinity for calcite [17]. The aim of this work described here is to investigate the effect of molecular adsorption of seven phosphonates (AMP, NDP, ATMP, EDTMP, TDTMP, HDTMP and DTPMP) on the four stepped surfaces of calcite using the same method in an alkalescent industrial water environment. AMP, NDP and ATMP have separately one, two and three 
phosphonate groups connected by a nitrogen atom. EDTMP, TDTMP, and HDTMP have four phosphonate functional groups attached to amine nitrogen atoms through methylene chain. The only difference is the length of the backbone methylene chain. But DTPMP has five phosphonate groups attached to three $\mathrm{N}$ atoms. The structures (seen in Fig.1) are protonated referring to the $\mathrm{pH}$ values in the range of 7 8 in an alkalescent industrial water environment.

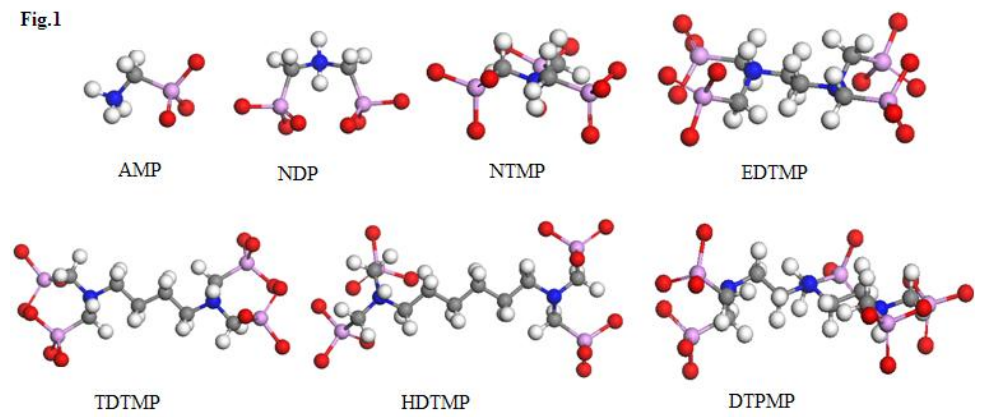

Fig. 1 The structures of seven protonated phosphonates

\section{Method}

We have simulated seven protonated phosphonates (AMP DTPMP) onto the four stepped calcite (104) surfaces using Materials Studio v3.0 (MS) under compass force field used in the early studies. In this paper, the dielectric constant $(\varepsilon)$ and the temperature are separately set of 78.0 and $350 \mathrm{~K}$ according with the actual circular water environment.

\section{Results and Discussion}

\section{Calcite (104) Stepped Surfaces}

Calcite has a rhomobohedral crystal structure with space group $\mathrm{R} / 3 \mathrm{C}$ and its lattice parameters are $a=b=4.99 \AA, c=17.06 \AA, \alpha=\beta=90.0^{\circ}, \gamma=120.0^{\circ}$. As the (104) cleavage surface is by far the most stable plane, both theoretically and experimentally, it dominates the growth morphology of calcite [18] Stepped surfaces are important in crystal growth, according to the periodic bond chains (PBC) theory. Crystal growth morphologies are grouped into three types, namely flat, step and kink [19]. Flat face is the most stable surface due to the slowest growth rate, whereas the growth rate of step face is larger because of the stronger adsorbability. Kink faces rapidly disappear on account of the fastest growth rate. That also indicates the adsorption on stepped faces is the most remarkable in the presence of organic additives and the optimal binding sites are stepped edge and corner.
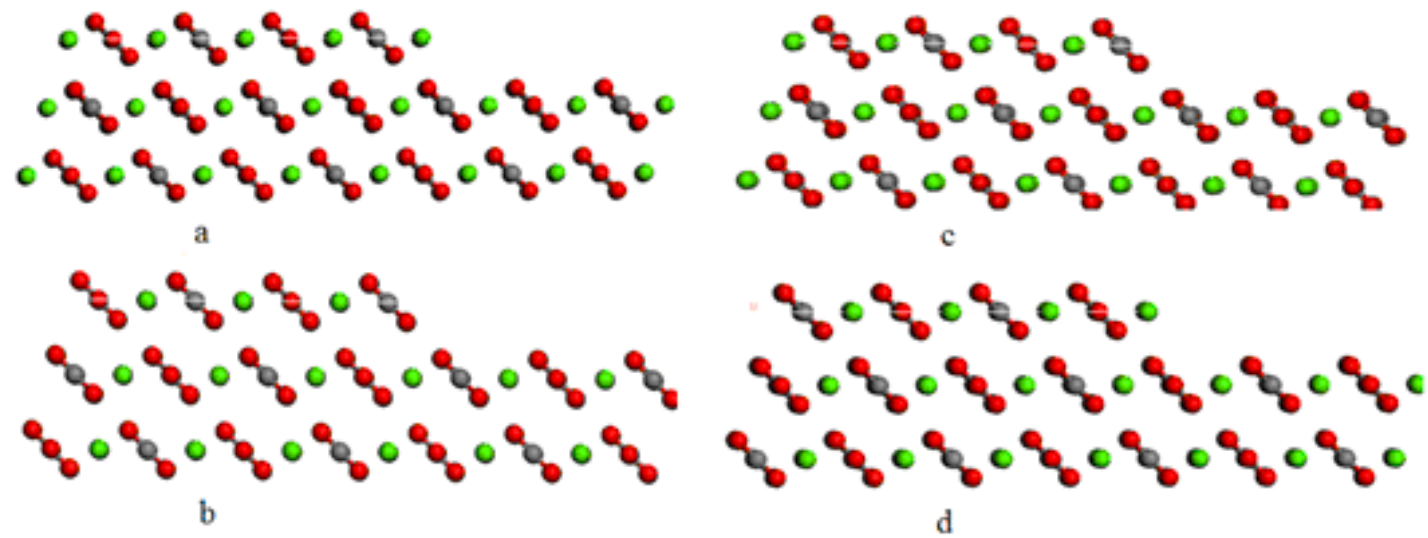

Fig. 2 Side view of calcite (104) stepped surfaces:(a) terminated by calcium ions, (b) terminated by carbonate groups, (c) terminated by calcium ions on the left edge and carbonate groups on the right edge, (d) terminates by calcium ions and carbonate groups in the opposite direction. 
In this study, the (104) plane is divided into four different stepped surfaces, terminated by either calcium ions or carbonate groups (Fig. 2). One surface is terminated by calcium ions on both left and right edges, namely Ca step surface (Fig. 2a), whereas the second surface is terminated by carbonate groups $\left(\mathrm{CO}_{3}\right.$ step surface in Fig. $\left.2 \mathrm{~b}\right)$. The other two surfaces are both terminated by calcium ions and carbonate groups, while the only difference is in the opposite direction $\left(\mathrm{Ca}-\mathrm{CO}_{3}\right.$ step surface in Fig. 2c, $\mathrm{CO}_{3}$ - Ca step surface in Fig. 2d).

\section{Adsorption of Seven Phosphonates on Calcite (104) Stepped Surfaces}

We separately considered the adsorption of seven different organic molecules (AMP DTPMP) on the various calcite surfaces. The results show that all of the phosphate groups point towards the calcite stepped surface, nevertheless the phosphate group in AMP points away from the surface. If phosphonate molecules have apparently adsorption effect, they must contain at least two phosphate groups. The result suggests that the phosphonates interact well with the surface on account of its oxygen atoms of phosphate group contacting well with calcium ions of the surface, and the formation of hydrogen bond also enhances the strength of interaction of the phosphonates with the surface. In addition, it is obviously found that all phosphonate molecules are combined at the stepped edge site according with PBC theory.

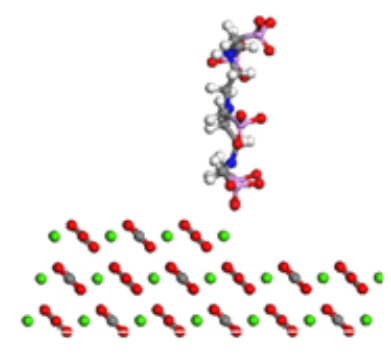

a

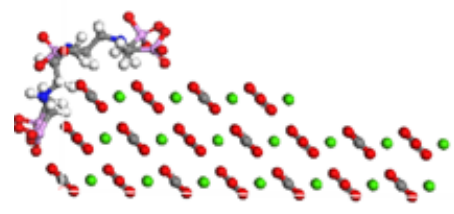

C

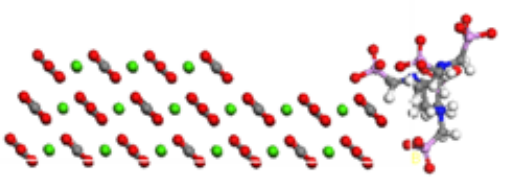

$\mathrm{b}$

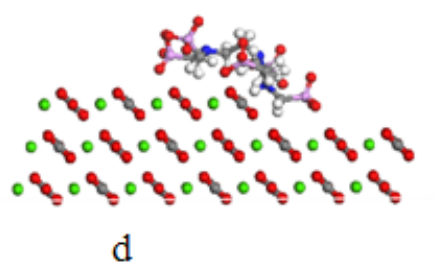

Fig. 4 The adsorption of DTPMP on the four calcite (104) stepped surfaces

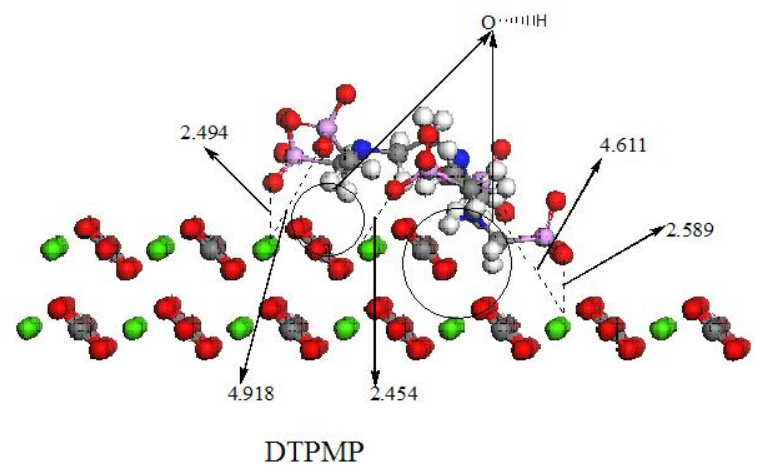

Fig. 5 The interaction mode of DTPMP on the Ca-CO3 step surface

Fig. 3 displays the adsorption of DTPMP on the four calcite stepped surfaces. The image is shown in side view in order to clearly indicate whether the phosphonate groups close to the surfaces. It shows that DTPMP interact well with the four stepped surfaces at the stepped edge sites, although the optimal binding sites are different. For example, Fig. 4 shows the detailed coordination mode of DTPMP on the $\mathrm{Ca}-\mathrm{CO}_{3}$ step surface. It is distinctly observed that the five phosphonate groups in DTPMP "approach" the crystal surface. The Ca-O (P) bond distances are separately $2.494 \AA$, 2.454 
A, $2.589 \AA$ A 4.611 and $4.918 \AA$. Three distances are close to $2.5 \AA$ in accordance with the Ca-O bond length in experience. Moreover, the hydrogen atoms in DTPMP and the oxygen atoms in carbonate group form weak hydrogen bond interactions.

For further analysis of the effectiveness of inhibition, we consider the binding energy of interaction between the seven phosphonates ligands (AMP DTPMP) and the calcite surfaces listed in Tab. 1. The energy of interaction is dominated by electrostatic interaction, and the van der Waals and hydrogen bond interaction play a very small role. Based on the data in Tab. 1, the interaction energy is exothermic and DTPMP > HDTMP > TDTMP > EDTMP > NTMP > NDP > AMP, which can help us predict the effectiveness of scale inhibition. The result clearly indicates the importance of the number of phosphonate groups and the length of the backbone methylene chain connecting the two $\mathrm{N}$ atoms. When the number of phosphonate groups is larger than three, the binding energy is far more than the other three phosphonate acids. AMP has the weakest interaction with calcite due to the only one phosphonate group. EDTMP, TDTMP and HDTMP have four phosphonate functional groups attached to amine $\mathrm{N}$ atoms. More importantly, the inhibition potency varies with the length of alkyl chain connecting the two $\mathrm{N}$ atoms, which induces the effect: HDTMP > TDTMP > EDTMP. We suspect that the longer alkyl chains reduce the steric hindrance. DTPMP appears to be most potent than the others due to five phosphonate groups dominant in the interaction of phosphonate acids with calcite.

Tab. 1 Binding energies of seven compounds with the four calcite (104) stepped surfaces (unit $\mathrm{kcal} / \mathrm{mol}$ )

\begin{tabular}{cccccc}
\hline $\begin{array}{c}\Delta \mathrm{E} \\
\text { Molecules }\end{array}$ & $\mathrm{Ca}$ & $\mathrm{CO}_{3}$ & $\mathrm{Ca}-\mathrm{CO}_{3}$ & $\mathrm{CO}_{3}-\mathrm{Ca}$ & $E_{\text {bind,cor }}$ \\
\hline AMP & -237.5 & -192.9 & -288.9 & -473.3 & -298.1 \\
NDP & -257.5 & -223.1 & -316.1 & -528.0 & -331.2 \\
NTMP & -264.5 & -234.1 & -308.2 & -573.4 & -345.0 \\
EDTMP & -294.7 & -804.6 & -325.2 & -723.4 & -537.0 \\
TDTMP & -345.4 & -838.3 & -327.3 & -823.2 & -583.6 \\
HDTMP & -368.4 & -853.7 & -366.2 & -808.1 & -599.1 \\
DTPMP & -375.1 & -933.8 & -376.5 & -831.3 & -629.2 \\
\hline
\end{tabular}

\section{Conclusion}

This paper is a study of molecular interaction of phosphonate acids with the four calcite (104) stepped surfaces using molecular dynamic simulation. In summary, the presence of organic phosphonates has a significant inhibition effect on precipitation of calcite. The results suggest that the step edge and the corner are optimal binding sites. The binding energies have proved that the conclusion that the inhibition of organic phosphonates is DTPMP > HDTMP > TDTMP > EDTMP > NTMP > NDP > AMP according to their phosphonate group number and the length of the backbone alkyl chain. Future work will focus on studies of the interaction of phosphonates with the calcite stepped surfaces in presence of water or $\mathrm{Na}^{+}$and $\mathrm{Zn}^{2+}$.

\section{Acknowledgments}

This work is supported by Institute of Industrial Chemistry, Nanjing University of Science \& Technology and School of Metallurgy and Materials Engineering, Jiangsu University of Science and Technology.

\section{References}

[1] B. J. Alder, T. E. Wainwright, Phase Transition for a Hard Sphere System, Chem. Phys. 27(1957) 1208-1209. 
[2] N. Bernd , T. S. Alan, Adsorption of Phosphonates onto the Goethite- Water Interface, J. Colloid Interface Sci. 214(1999) 20-30.

[3] L. A. Bromley, D. Cottier, R. J. Davey, Interactions at the organic/ inorganic interface: molecular design of crystallization inhibitors for barite, Langmuir. 12(9) (1993) 3594.

[4] C. Y. Chen, M. Z. Xia, F. Y. Wang, Influence of three Organic Phosphonates on Calcite Crystal Growth, Advanced Materials Research, 154- 155 (2010) 437-442.

[5] J. Franca, R. R.Willian, L. R. Andrew, Molecular Modeling of Phosphonate Molecules onto Barium Sulfate Terraced Surfaces. J. Phys. Chem. B, 110(2006) 7414-7424.

[6] A. J.Gratz, P. E. Hillner, P. K. Hansma, Step Dynamics and Spiral Growth on Calcite. Geochim. Cosmochim. Acta, 57(1993) 491-495.

[7] Y. S. Han, G. Hadiko, M. Fuji, M. Takahashi, Crystallization and Transformation of Vaterite at Controlled pH. J. Cryst. Growth, 289 (2006) 269-274.

[8] P. Hartman, W. G. Perdok, On the Relations between Structure and Morphology of Crystals. Acta Crystallngraphica, 8(1955) 49-52.

[9] F. Manoli, E. Dalas, Calcium Carbonate Crystallization in the Presence of Glutamic Acid. J. Cryst. Growth, 222(2001) 293-296.

[10] F. Manoli, J. Kanakis, P. Malkaj, E. Dalas, The Effect of Aminoacids on the Crystal Growth of Calcium Carbonate. J. Cryst. Growth, 236(2002) 363-370.

[11] H. de L.Nora, C. P. Stephen, Atomistic simulation of the effect of molecular adsorption of water on surface structure and energies of calcite surfaces. J. Chem. Soc, 93(3) (2004) 467- 475.

[12] K. I. Parsiegla, J. L. Katz, Calcite Growth Inhibition by Cooper( II ) -An Introduction, Holt. J. Cryst. Growth, 200(1999) 213-226.

[13] B. R. Pradip, T. K. Rao, Molecular Modeling of Interactions of Diphosphonic Acid Based Surfactants with Calcium Minerals. Langmuir, 18(2002) 932-940.

[14] M. M. Reddy, A. R. Hoch, Calcite Crystal Growth Rate Inhibition by Polycarboxylic Acid. J. Colloid Interface Sci., 236(2001) 365-370.

[15] S. L. S. Stipp, W. Gutmannsbauer, T. Lehrmann, The Dynamic Nature of Calcite Surfaces in Air. Am. Minera, 81(1996), 1-8.

[16]Sun, H. (1998) Compass: An ab Initio Force Field Optimized for Condensed-Phase Application, Overview with Detail on Alkane and Benzene Compounds. Phys. Chem. B, 102, 7338-7364.

[17] C. Y. Chen, W. Lei, M. Z. Xia, F. Y. Wang, X. D. Gong, Modeling of several phosphonates onto the stepped calcite (011) surface, Desalination, 309(2013) 208- 212.

[18] G. C. Timothy, H. de L. Nora, A Computer Modeling Study of the Competitive Adsorption of Water and Organic Surfactants at Surfaces of the Mineral Scheelite, Langmuir, 20 (2004) 3984-3994.

[19]Westin, K. J., Rasmuson, A. C. (2005) Crystal Growth of Aragonite and Calcite in Presence of Citric Acid, DTPA, EDTA and Pyromellitic Acid. J. Colloid Interface Sci., 282, 359-369. 